\title{
Maintenance Treatment with Fluoxetine is Necessary to Sustain Normal Levels of Synaptic Markers in an Experimental Model of Depression: Correlation with Behavioral Response
}

\author{
Analía Reinés*,1,2, Marina Cereseto', Alejandro Ferrero', Laura Sifonios', Maria Fernanda Podestá' \\ and Silvia Wikinski ${ }^{1,3}$ \\ I'Instituto de Investigaciones Farmacológicas (ININFA), CONICET, Universidad de Buenos Aires, Buenos Aires, Argentina; ${ }^{2}$ Cátedra de \\ Farmacología, Facultad de Farmacia y Bioquímica, Universidad de Buenos Aires, Buenos Aires, Argentina; ${ }^{3} 1{ }^{\circ}$ Cátedra de Farmacología, Facultad \\ de Medicina, Universidad de Buenos Aires, Buenos Aires, Argentina
}

\begin{abstract}
Dysfunction of hippocampal plasticity has been proposed to play a critical role in the pathophysiology of depression. However, antidepressant drug effects on synaptic plasticity and cytoskeletal remodeling remain controversial. The aim of the present study was to evaluate in animals exposed to the learned helplessness $(\mathrm{LH})$ paradigm, an accepted experimental model of depression, the effect of chronic treatment with fluoxetine (FLX) on synaptic and cytoskeletal proteins known to undergo plastic changes. Synaptophysin (SYN), postsynaptic density 95 (PSD-95), axon growth-associated protein 43 (GAP-43), and cytoskeletal proteins (intermediate neurofilaments and MAP-2) were studied in the hippocampus by immunohistochemistry. Whereas LH animals treated 21 days with saline (LH-S group) displayed diminished SYN and PSD-95 immunostainings in the CA3 but not in the DG, chronic treatment with FLX not only reversed the despaired behavior induced by exposure to LH paradigm, but also fully recovered SYN and PSD-95 labeling to control values. Similar results were obtained for the axonal remodeling marker GAP-43. FLX treatment did not modify either the decreased light neurofilament subunit (NFL) observed in the hippocampus of LH animals or any other cytoskeletal protein studied. When FLX treatment was withdrawn for 90 days in those LH-FLX animals in which reversion of despair had been observed at day 25, recurrence of despaired behavior was found accompanied by decreased SYN, PSD-95, and NFL labelings. Results indicate that the synapse remodeling induced by FLX in the CA3 region could underlie its behavioral efficacy despite the absence of cytoskeletal remodeling and that the stability of synaptic changes would depend on the continuous administration of the drug.

Neuropsychopharmacology (2008) 33, 1896-1908; doi:10.1038/sj.npp.1301596; published online 17 October 2007
\end{abstract}

Keywords: hippocampal plasticity; synaptic proteins; neurobiology of depression; recurrence of depression; antidepressant; cytoskeleton

\section{INTRODUCTION}

Major depression is a highly prevalent disorder, affecting more than 121 million people all over the world (World Health Organization, 2001). Besides the observation that placebo effect seems to significantly contribute to the antidepressant response (Posternak and Zimmerman, 2007) and that 3-4 weeks are needed to obtain therapeutic results; over the past years, it became clear that almost $40 \%$ of patients fail to obtain benefit of a well-conducted treatment (Nemeroff, 2007). Finally, current therapeutic strategies seem not to consistently modify the natural course of the

* Correspondence: Dr A Reinés, Instituto de Investigaciones Farmacológicas (ININFA), CONICET, Universidad de Buenos Aires, Junín 956 5to. Piso, Buenos Aires CI I I 3 AAD, Argentina,

Tel: 54 || 496| 5949, Fax: 54 || 4963 8593,

E-mail: areines@ffyb.uba.ar

Received I May 2007; revised II September 2007; accepted 12 September 2007 illness, since recurrence rates are over 50\%, making recurrence the rule rather than the exception in unipolar depression (Keller, 2001). It has been shown that each affective episode carries higher risk of recurrence than the previous one.

Neuronal plasticity or remodeling is a critical process that underlies the normal central nervous system function by which the brain acquires information and makes the appropriate adaptive responses in future-related settings. Currently, depression may be considered a consequence of stress-induced impairment in the plasticity of several brain areas (Duman, 2002). In particular, the hippocampus, a region critically involved in motivation and emotion processing (Eichenbaum and Otto, 1992), has been shown affected in depressed patients (Bremner et al, 2000; Mervaala et al, 2002; Sheline et al, 2000) and in animals exposed to experimental models of depression (Watanabe et al, 1992; Magariños et al, 1996). Dysfunction of neuronal plasticity could therefore contribute to the pathophysiology 
of mood disorders and recovery could occur by induction of the appropriate plasticity or remodeling phenomena (Akhondzadeh, 1999; Duman, 2002).

Several magnetic resonance imaging (MRI) studies of patients with major depression have described a diminution of hippocampal volume (Bremner et al, 2000; Mervaala et al, 2002; Sheline et al, 2000). In addition, some phenomena possibly related to this volume diminution have been reported in preclinical studies that employ stress exposure as a predisposing factor to depression: shrinkage of the apical tree of hippocampal CA3 pyramidal neurons (Watanabe et al, 1992; Magariños et al, 1996), alterations in axonal components, downregulation of neurogenesis in the subgranular layer of the adult dentate gyrus and putative glial changes (Czéh and Lucassen, 2007). Synapses have also been found to underlie functional and morphological changes under stress conditions. It is known that chronic stress induces structural remodeling of excitatory axo-spinous synaptic connectivity (Donohue et al, 2006) and differentially regulates synaptic protein expression in the hippocampus (Thome et al, 2001; Rosenbrock et al, 2005).

Regarding the antidepressant action on these plastic phenomena, a well-established effect is to stimulate the synthesis of neurotrophic factors (Nibuya et al, 1995, 1996; Chen et al, 2001) and to promote neurogenesis in the hippocampus (Malberg et al, 2000). On the contrary, the effect of antidepressant treatment on cytoskeletal and synaptic plasticity remains controversial. For instance, despite Czéh et al (2001) have shown that the atypical antidepressant tianeptine prevents the hippocampal volume loss induced by stress and Magariños et al (1999) have proved tianeptine efficacy to reverse stress-induced rat hippocampal atrophy visualized by Golgi staining, fluoxetine (FLX) has not been effective on these cytoskeletal changes (Magariños et al, 1999). In coincidence with these preclinical reports, clinical studies have not been able to confirm that antidepressant treatment reverses the hippocampal volume diminution found in depressed patients (Neumeister et al, 2005). As plasticity-responsive elements, synaptic proteins have been shown to differentially respond to antidepressant drugs. Indeed, whereas venlafaxine normalizes, imipramine has no effect on the stress-induced decrement of synaptic proteins ( $\mathrm{Xu}$ et al, 2004; Iwata et al, 2006). It is worth noticing that despite the evidence indicates that antidepressants might modulate some components of cytoskeletal and synaptic plasticity, it remains to be further clarified whether these effects are related to the antidepressant behavioral efficacy.

So, the aim of this work was to examine the effect of the chronic treatment with the antidepressant FLX on parameters linked to neuronal plasticity in animals exposed to the learned helplessness (LH) paradigm, an accepted experimental model of depression. Presynaptic (synaptophysin (SYN)) and postsynaptic (postsynaptic density 95 (PSD-95)) proteins, axon growth-associated protein 43 (GAP-43) and cytoskeletal proteins (intermediate neurofilaments and microtubule-associated protein 2 (MAP-2)) were used as markers to study neuronal plasticity in the hippocampus.

Several experimental models of depression are currently available. Most of them are based on apparent similarities between clinical features and experimentally induced behavioral impairments in animals. It is important to note that hopelessness and subjective suffering are major features of depression (Markowitz and Milrod, 2005), and subsequently animal models have both theoretical and practical limitations. For our work, we have chosen an experimental model of depression in which behavioral and structural (i.e. hippocampal) alterations last for long periods of time. Therefore, we were able to examine the relationship between the plastic processes induced by FLX and its behavioral efficacy as well as the persistence of the antidepressant-induced changes once the treatment was interrupted, a methodological approach thought to mimic the actual condition in the clinical setting. By taking this approach, we have been able to show that synapse remodeling might be a target for the antidepressant action and that the stability of synaptic changes would depend on the continuous administration of the drug.

\section{MATERIALS AND METHODS}

\section{Animals and Drugs}

Male Wistar rats weighing 200-230 g at the beginning of the experiments were used. Animals were purchased at the Bioterio Central, Facultad de Ciencias Exactas y Naturales, Universidad de Buenos Aires, Buenos Aires, Argentina. Rats were housed in groups of four in an air-conditioned and light-controlled room (temperature: $20 \pm 2{ }^{\circ} \mathrm{C}$, light phase: 0800-2000 hours). Food and water were given ad libitum. Experiments were all carried out in accordance with the Guide for Care and Use of Laboratory Animals provided by the National Institute of Health, USA. Special care was taken to minimize the number of animals used and their suffering. FLX was kindly provided by Laboratorios Gador (Argentina). All chemical substances were of analytical grade. Mouse monoclonal antibodies anti-light neurofilament subunit (NFL), anti-medium neurofilament subunit (NFM), antiheavy neurofilament subunit (NFH), anti-MAP-2, anti-SYN, and anti-GAP-43, secondary biotinylated antibody and streptavidin complex used for immunohistochemical studies were purchased from Sigma Chemical Co. (St Louis, MO, USA). Mouse monoclonal antibody anti-PSD-95 was purchased from Affinity BioReagents Inc. (Golden, CO, USA). FITC-labeled secondary antibody was purchased from Jackson ImmunoResearch Laboratories Inc. (West Grove, PA, USA). Vectashield mounting medium for fluorescence microscopy was purchased from Vector Laboratories Inc. (Burlingame, CA, USA).

\section{LH Paradigm in Rats}

LH induction (training session). The LH paradigm was performed as previously described (Reinés et al, 2004) according to Nakagawa et al (1999) and Vollmayr and Henn (2001) with modifications. Rats were randomly assigned to LH or control (C) groups. Animals were individually placed in the treatment chamber that consisted of a $28 \times 21 \times 25 \mathrm{~cm}^{3}$ box with brown Plexiglas walls and equipped with a stainless-steel grid floor. A constantcurrent shocking device was used to deliver 60 inescapable foot shocks $(0.6 \mathrm{~mA})$ for $15 \mathrm{~s}$ every $\mathrm{min}$ (i.e. for $1 \mathrm{~h}$ ). Control 
rats were placed on the grid in identical chambers for $1 \mathrm{~h}$, without receiving the uncontrollable electric shocks. After this training session, animals were replaced in their own cages in groups of four (same condition as before the session). All the helplessness induction trials were performed in the morning.

LH behavioral test (test session). Quantification of escape deficit, which allowed the identification of animals that displayed the despaired behavior, was performed as previously described (Reinés et al, 2004) according to Nakagawa et al (1999) and Vollmayr and Henn (2001) with modifications. Briefly, both LH and control animals were subjected to an avoidance task in a shuttle box which consisted of two equal-sized compartments divided by a Plexiglas partition fitted out with an opening $(7 \times 7 \mathrm{~cm})$ and with a grid floor consisting of stainless-steel rods placed $1-\mathrm{cm}$ apart. Animals were placed individually into the shuttle box and allowed to habituate to the environment for 5 min. Following this exploration session, 15 stimulus-shock trials were presented for a period of $15 \mathrm{~min}$, that is one trial per min. In each trial, the shock duration was $20 \mathrm{~s}$ with an intertrial time of $40 \mathrm{~s}$. In the first five trials, the door was opened immediately after starting the shock and in the other 10 trials there was a 4-s delay period after starting the shock. The time latency to change side during the 20-s shock on was recorded as the way to evaluate the LH behavior (see Anisman and Merali (2001) for details about the appropriateness of the test). In the described conditions, approximately $50 \%$ of shocked rats displayed despaired behavior with mean escape latency of $15 \mathrm{~s}$ or higher; only these animals were finally assigned to the LH group.

\section{Experimental Design}

Evaluation of the relationship between FLX behavioral effects and parameters of neuronal plasticity in the hippocampus at day 25. Animals in which despaired behavior was observed in the test session performed 4 days after the training session (mean escape latency recorded in the 15 trials was $15 \mathrm{~s}$ or higher) were randomly divided into two groups: LH-FLX (injected with $10 \mathrm{mg} / \mathrm{kg}$ of FLX, i.p. for 21 days) or LH-S (injected with saline for 21 days). Similarly, control (C) animals (rats that received no shock during the training session), tested on day 4 were subdivided into two groups, one was injected with saline for 21 days (C-S), and the other received $10 \mathrm{mg} / \mathrm{kg}$ of FLX, i.p. for the same period of time (C-FLX). Each experimental group consisted of five to six animals. All injections were administered during the mornings. On day 25 a test session took place, and $1 \mathrm{~h}$ later animals were killed to perform immunohistochemical assays (Figure 1a). Animals were weighed at the beginning of the experiments and thereafter on a weekly basis. We observed that exposure to inescapable stress did not have any effect on the body weight. Results in grams, expressed as the mean $\pm S D$ of the differences between the first and the last measurements were as follows: C-S $68 \pm 30$ and LH-S $58 \pm 25$. On the other hand, FLX treatment decreased the rate of body weight increment both in $\mathrm{C}$ and in LH groups, as shown by the following values: C-FLX $1 \pm 24 \mathrm{~g}$ and LH-FLX $11 \pm 24 \mathrm{~g}$. When comparison among the four groups was performed, a
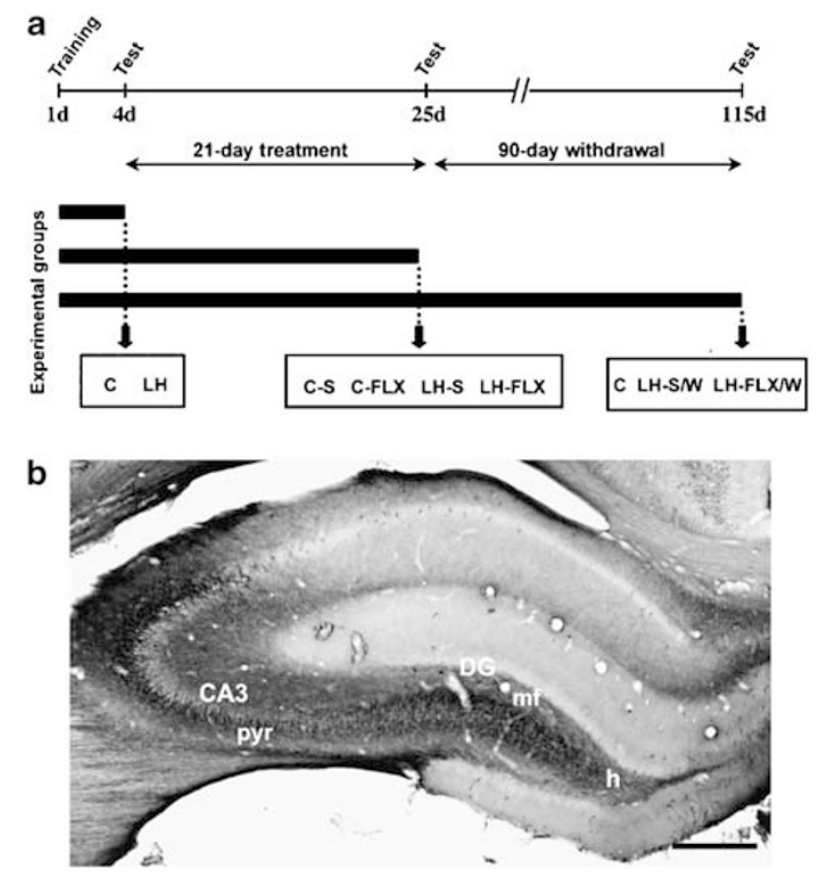

Figure I Experimental design and anatomical localization of hippocampal areas used for the immunohistochemical studies. The schematic representation of pharmacological treatments shows the experimental groups at 4, 25, and I 15 days after the training session. Animals subjected to the learned helplessness paradigm that developed the depressive-like behavior correspond to learned helplessness $(\mathrm{LH})$ group. Control animals ( $C$ groups) are those nonshocked but injected $2 \mathrm{l}$ days with saline $(\mathrm{C}-\mathrm{S}$ ) or fluoxetine $(F L X)(10 \mathrm{mg} / \mathrm{kg}$, i.p.) (C-FLX) and tested at the indicated time points. At day 25, LH-S and LH-FLX groups are $\mathrm{LH}$ animals treated for $2 \mathrm{I}$ days with saline or FLX (10 mg/kg, i.p.), respectively. At II 5 day, LH-SM and $\mathrm{LH}-\mathrm{FL} X \mathrm{~W}$ groups correspond to $\mathrm{LH}$ rats treated for 21 days with saline or FLX, respectively, and whose pharmacological treatments were withdrawn for 90 days. Similarly, control animals at day I I 5 (C group) are those nonshocked but injected 21 days with saline and kept in standard conditions thereafter (a). The bright field photograph shows a 60 - $\mu$ m-thick section of the hippocampus immunostained for heavy neurofilament subunit (NFH), corresponding to plates 32-34 (from Bregma -3.14 to $-3.60 \mathrm{~mm}$ ) of Paxinos and Watson (1986) atlas. The staining allows observing CA3 and DG regions employed for immunohistochemical analysis (b). pyr, pyramidal cell layer; DG, dentate gyrus; h, hilus; mf, mossy fibers. Scale bar $=110 \mu \mathrm{m}$.

significant difference was detected between saline and FLXtreated groups $(P<0.001$, two-way ANOVA followed by Bonferroni test).

Evaluation of the long-term behavioral and structural consequences of interrupting the pharmacological treatment. In a new set of animals trained and tested as described above (C-S, LH-S, and LH-FLX), the pharmacological treatment was withdrawn after the test session on day 25. Only the LH-FLX animals in which reversion of despaired behavior was observed as escape latency of $6 \pm 1 \mathrm{~s}$ (LH-FLX/W group; $P>0.05$ vs control animals) were included in the study ( $50 \%$ of total LH-FLX animals). At the same time, saline administration was withdrawn in control and LH-S rats (C and LH-S/W groups, respectively). Animals were maintained under standard conditions for 90 days. Each experimental group consisted of five to six animals. On day 115, animals were tested in the $\mathrm{LH}$ paradigm and $1 \mathrm{~h}$ later were killed as previously indicated to 
perform the immunohistochemical experiments (Figure 1a). The 90-day washout period ensured the complete elimination of FLX whose half-life is $53 \mathrm{~h}$ (Hardman and Limbird, 2001).

\section{Immunohistochemistry}

Tissue fixation. Similarly as reported elsewhere (Reinés et al, 2004; Ferrero et al, 2007), $1 \mathrm{~h}$ after the corresponding LH test session, animals were deeply anesthetized $(50 \mathrm{mg} / \mathrm{kg}$ ketamine hydrochloride and $2 \mathrm{mg} / \mathrm{kg}$ xylazine, i.p.) and perfused through the left ventricle with $4 \%$ paraformaldehyde. Brains were quickly removed and kept in the same cold fixative solution for $3 \mathrm{~h}$. After that, brains were washed three times in cold $0.1 \mathrm{M}$ phosphate buffer, $\mathrm{pH}$ 7.4, containing $5 \% \mathrm{w} / \mathrm{v}$ sucrose, and left in this washing solution for $18 \mathrm{~h}$ at $4^{\circ} \mathrm{C}$. Coronal $60 \mu \mathrm{m}$ thick brain sections were cut using a vibratome and stored at $-20^{\circ} \mathrm{C}$.

Staining procedure. Brain sections were processed in freefloating state as previously described (Reinés et al, 2004). After inhibition of endogenous peroxidase activity, brain sections were blocked with $3 \% \mathrm{v} / \mathrm{v}$ normal ovine serum and incubated for $48 \mathrm{~h}$ at $4{ }^{\circ} \mathrm{C}$ with one of the following primary monoclonal antibodies against NFL, NFM, NFH, or MAP-2, diluted $1: 1000$ and $1: 2000 \mathrm{v} / \mathrm{v}$ for NFs and MAP-2, respectively. Sections were then incubated for $1 \mathrm{~h}$ at room temperature with the biotinylated secondary antibody $(1: 100)$ followed by another $1 \mathrm{~h}$ incubation period with streptavidin-peroxidase complex solution diluted $1: 200$. Finally, peroxidase activity was developed by adding $0.035 \%$ $\mathrm{w} / \mathrm{v} 3,3^{\prime}$-diaminobenzidine plus $2.5 \% \mathrm{w} / \mathrm{v}$ nickel ammonium sulfate and $0.1 \% \mathrm{v} / \mathrm{v}_{2} \mathrm{O}_{2}$. Sections were mounted onto gelatin-coated slides, dehydrated and coverslipped using Permount for light microscope observation. Specificity of immunohistochemical procedure was assessed by omitting primary antibodies. In all cases, no immunohistochemical labeling was observed.

Immunofluorescence procedure. Briefly, free-floating brain sections were blocked during $1 \mathrm{~h}$ with $3 \% \mathrm{v} / \mathrm{v}$ normal goat serum in phosphate-buffered saline (PBS) and incubated for $48 \mathrm{~h}$ at $4^{\circ} \mathrm{C}$ with one of the following primary monoclonal antibodies against SYN, PSD-95, or GAP-43 diluted $1: 1000$ $\mathrm{v} / \mathrm{v}$. After thoroughly washed, sections were incubated with FITC-labeled secondary antibody for $1 \mathrm{~h}$ at room temperature and mounted onto gelatin-coated slices using mounting medium for fluorescence microscope observation. Specificity of immunohistochemical procedure was assessed by omitting primary antibodies that gave no immunohistochemical labeling.

\section{Morphometric Measurement}

Image acquisition. FITC-labeled SYN, PSD-95, and GAP-43 structures from CA3 and DG areas of hippocampus were captured employing a 450-490 nm excitation filter in an Eclipse 50i Nikon epi-fluorescence microscope equipped with a Nikon DS-5M cooled camera on line with Image J analyzer (NIH). Homogeneous illumination of the microscopic field was kept constant by ensuring the light source center positioning. To evaluate NFs and MAP-2 positive fibers, immunolabeled structures were captured employing an Axiophot Zeiss light microscope equipped with a video camera on line with a Zeiss-Kontron VIDAS image analyzer. Either for immunofluorescent or colorimetric labeling, the analog images were digitized into an array of $512 \times 512$ pixels corresponding to an area of $140 \times 140 \mu \mathrm{m}(40 \times$ primary magnification). The resolution of each pixel was 256 gray levels. Threshold setting was performed according to previous reports (Ramos et al, 2000; Tagliaferro et al, 2002; Reinés et al, 2004). For that purpose, a range of gray value (threshold value) was interactively selected to allow the segmentation of the specific signal from the background and thereafter was kept constant along each experiment. Plates containing scanned microphotographs were prepared with Adobe Photoshop 6.0 software (Adobe Systems Inc.).

Positive structure quantification. All measurements were performed on coded slides by different observers to ensure objectivity. Immunolabeled structures were measured in CA3 and hilar region of the DG of hippocampus (Figure 1b). Hippocampal areas were delimited according to plates 3234 (from Bregma -3.14 to $-3.60 \mathrm{~mm}$ ) of Paxinos and Watson (1986) atlas. Only tissue sections corresponding to these coordinates were included in the quantification, ensuring that the region of interest was equivalent among animals and experiments. To ensure the evaluation of similar fields inside the CA3 and the DG regions of different tissue sections, dorso-ventral and lateral references corresponding to the mentioned plates 32-34 of the atlas of Paxinos and Watson (1986) were employed. SYN and PSD95 positive structures were quantified as relative area (immunoreactive area/total area of the field of view) employing the Image $\mathrm{J}$ analyzer (NIH). In the case of GAP-43, only qualitative analysis was performed due to the dense labeling in the area of interest. To evaluate NFs and MAP-2 positive fibers, total area of immunolabeled fibers per field was measured employing a Zeiss-Kontron VIDAS image analyzer. Evaluation of neuronal projections labeled for cytoskeletal proteins was ensured by excluding cell bodies from measurements. In each section, immunoreactive area was recorded in three contiguous fields (without overlapping the measured fields) of CA3 and hilar region of the DG of left and right hippocampus.

\section{Data Analysis}

Escape latency is the mean value $( \pm S D)$ of five to six animals per group. Statistical significance of differences between control and LH animals were determined at day 4 by Mann-Whitney test or at days 25 and 115 by KruskalWallis test followed by Dunn's multiple comparison test.

Immunoreactive areas are mean values $( \pm S D)$ of three to four separate experiments. Individual experiments consisted of three to five tissue sections of each of the five to six animals per group. In each section, immunoreactive area was measured in three fields of the CA3 and hilar region of the DG of left and right hippocampus. Differences among mean immunoreactive areas or mean relative areas were statistically analyzed by one or two-way ANOVA followed by Bonferroni test.

The probability level indicative of statistical significance was set at $P<0.05$. 


\section{RESULTS}

\section{Chronic Treatment with FLX Corrects the Behavioral Deficit Observed in LH Animals}

Four days after the training session, the pharmacological treatment began in LH animals that showed increased escape latency indicative of despaired behavior $(P<0.01 \mathrm{vs}$ controls, Mann-Whitney test) (Figure 2a). Chronic treatment (21 days) with FLX induced no effect on control animals (C-FLX group) in comparison with saline-injected ones (C-S group) (Figure 2b). Chronic treatment with FLX of LH animals (LH-FLX group) significantly decreased the escape latency $(P<0.05$ vs LH-S group, Kruskal-Wallis test followed by Dunn's multiple comparison test) to reach a value that was not statistically different from that observed in control groups (Figure 2b).

The Behavioral Efficacy of FLX Treatment is Coincident with Synaptic Remodeling in the Hippocampal CA3 Region

Immunostainings for pre- and postsynaptic markers SYN and PSD-95, respectively, were analyzed in CA3 and DG regions of hippocampus.

In the apical dendritic arbor of CA3 pyramidal neurons of C-S animals, SYN labeling was higher in strata lucidum and
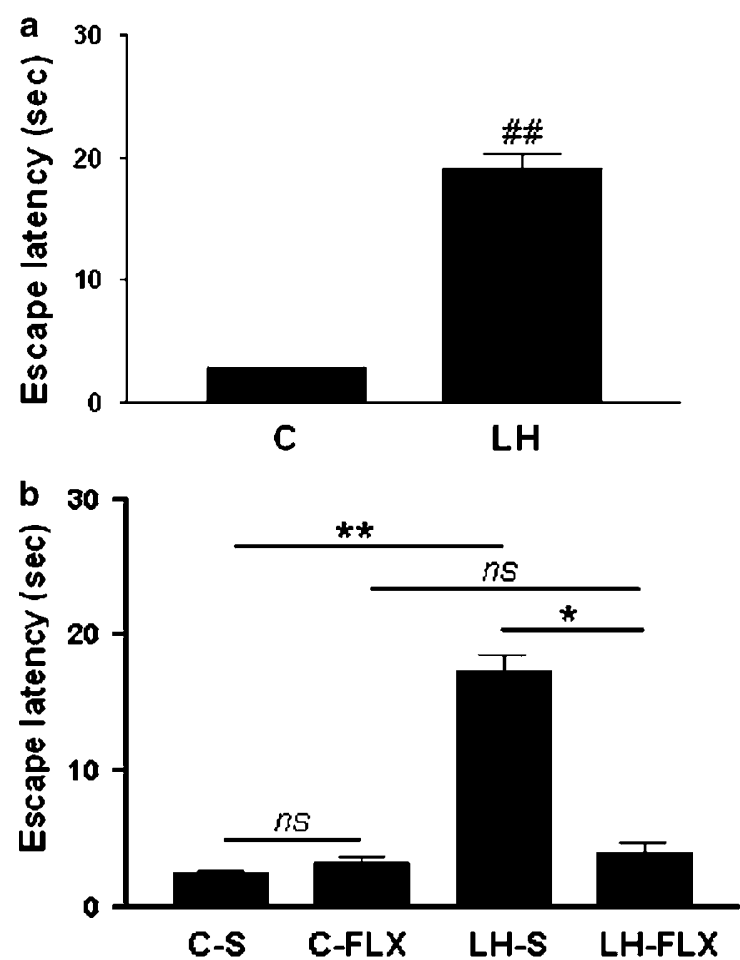

Figure 2 Fluoxetine (FLX) treatment corrects the behavioral deficit induced in learned helplessness ( $\mathrm{LH}$ ) animals. Four days after the training session, increased escape latency (sec) indicative of despaired behavior was observed in the LH group (a). Twenty one day treatment with FLX of learned helplessness animals ( $\mathrm{LH}-\mathrm{FLX}$ group) corrected the behavioral deficit as shown by the significant decrease in the escape latency (sec) that failed to differ from controls (C-S and C-FLX) (b). Data are expressed as mean values ( $\pm S D, n=5-6)$. ${ }^{\# \#} P<0.01$ vs control, Mann-Whitney test. $* P<0.05$; $* * P<0.01 ; \mathrm{ns}$, nonsignificant between bars, Kruskal-Wallis test. radiatum and lower in the pyramidal cell layer. As previously shown by $\mathrm{Li}$ et al (2002) and Grillo et al (2005), the immunostaining pattern for SYN was observed as bright dots that outline the dendrites. The same pattern was observed in control animals chronically treated with FLX (C-FLX). Whereas the immunostaining for SYN was found decreased in the CA3 region of the hippocampus of LH animals treated with saline for 21 days (LH-S), FLX treatment induced no effect on control animals (C-FLX) but promoted a large increment in the labeling of this protein in LH-FLX group (Figure 3a). Quantification of the immunolabeled area revealed a $65 \%$ reduction of SYN staining in the LH-S group $(P<0.001$ $v s$ controls, two-way ANOVA followed by Bonferroni test) that was fully reversed by FLX treatment $(P>0.05 v s$ controls, twoway ANOVA followed by Bonferroni test) (Figure 3c).

Similar results were found with the postsynaptic marker PSD-95, a protein that specifically labels excitatory synapses. PSD-95 immunoreactivity was found considerably more intense along dendrites from strata lucidum and radiatum of the CA3 region than that in somata of pyramidal cell layer of control rats treated with saline. As previously described by Hunt et al (1996) and Grillo et al (2005) employing fluorescence microscopy, and in contrast to the small discrete spots discriminated by confocal microscopy (Cho et al, 1992), PSD-95 immunostaining pattern seemed relatively uniform throughout dendrites. Whereas LH-S animals showed diminished PSD-95 immunostaining in the $\mathrm{CA} 3$ region of hippocampus, $\mathrm{LH}$ animals treated with FLX (LH-FLX) displayed PSD-95 labeled structures similar to FLX-treated controls (C-FLX) which failed to differ from control rats treated with saline (C-S) (Figure 3b). Quantification of the immunolabeled area revealed a 73\% reduction of PSD-95 staining in the LH-S group $(P<0.001$ vs controls, two-way ANOVA followed by Bonferroni test) that was fully reversed by FLX treatment $(P>0.05$ vs controls, two-way ANOVA) (Figure 3d).

By contrast, in the hilar region of the DG quantification of immunolabeled area of these pre- and postsynaptic markers showed that either SYN or PSD-95 were unchanged in LH-S animals when compared to controls $(P>0.05$, two-way ANOVA). Relative immunoreactive areas were as follows: for SYN, $0.321 \pm 0.031$ in C-S; $0.323 \pm 0.040$ in C-FLX; $0.328 \pm 0.029$ in LH-S, and $0.318 \pm 0.020$ in LH-FLX group. For PSD-95, $0.184 \pm 0.018$ in C-S; $0.194 \pm 0.018$ in C-FLX; $0.186 \pm 0.020$ in LH-S, and $0.189 \pm 0.018$ in LH-FLX group.

\section{LH Paradigm Effect on Axonal Remodeling is Modified by FLX Treatment}

In control animals (C-S), GAP-43 labeled axons suffering plastic remodeling particularly in stratum oriens, lucidum, and radiatum of the $\mathrm{CA} 3$ region of the hippocampus. On the contrary, cell bodies of pyramidal neurons were devoid of GAP-43 labeling (Figure 4a). High magnification of GAP-43 positive structures in stratum lucidum allowed the observation of abundant fibers transversally oriented with respect to the apical dendrites of pyramidal neurons (Figure 4a, inset). The same pattern was observed in control animals chronically treated with FLX (C-FLX) (Figure $4 \mathrm{~b}$ and inset).

Animals that developed despaired behavior and were treated for 21 days with saline (LH-S) showed lower GAP-43 immunostaining in stratum lucidum and radiatum of the 
a
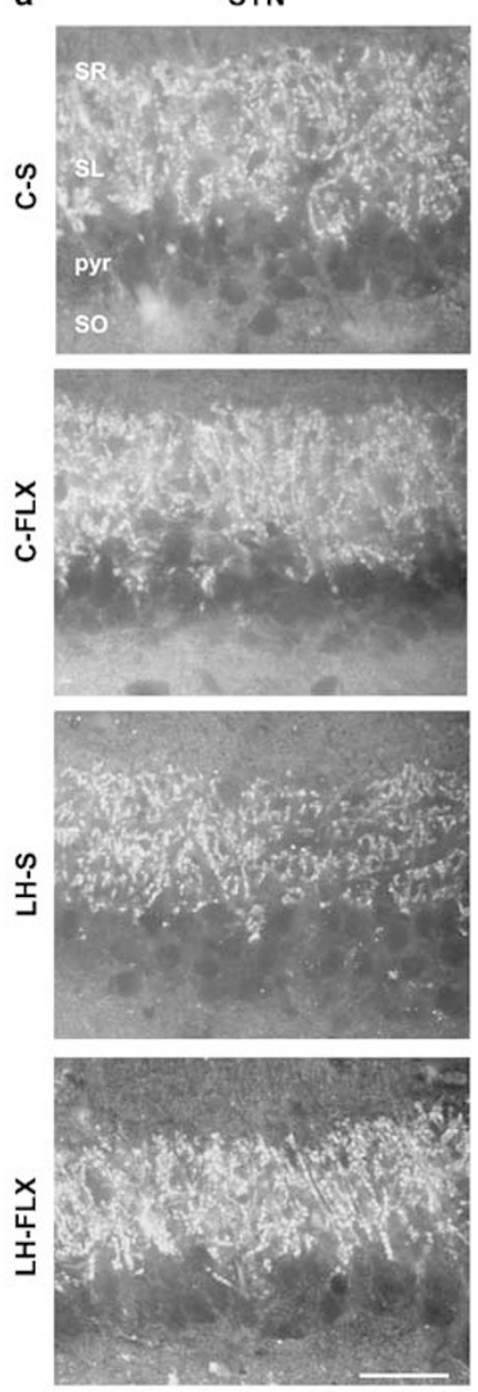

b
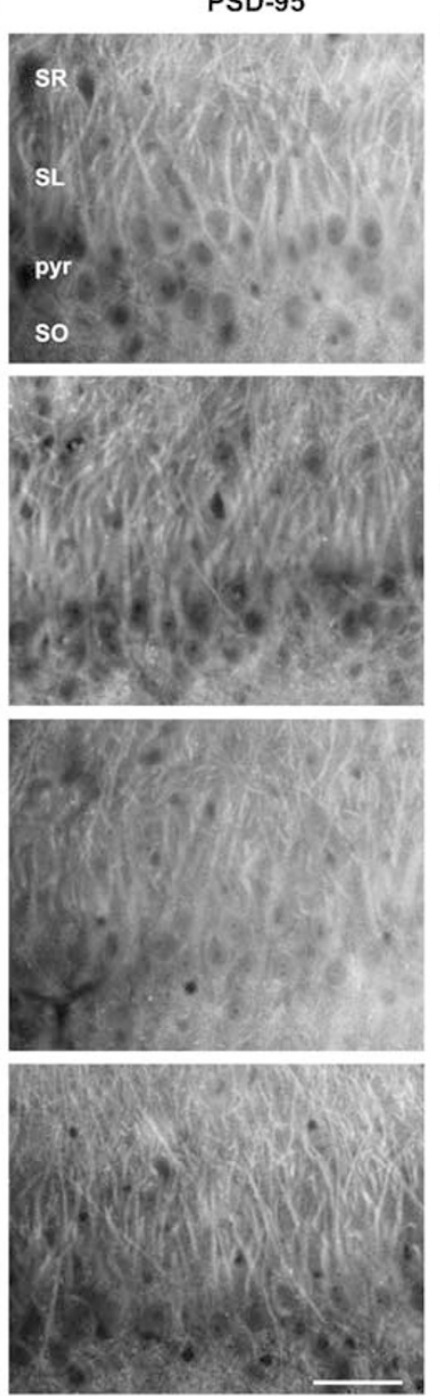

C

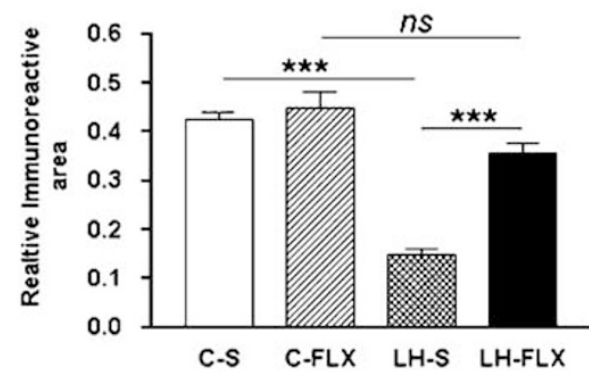

d

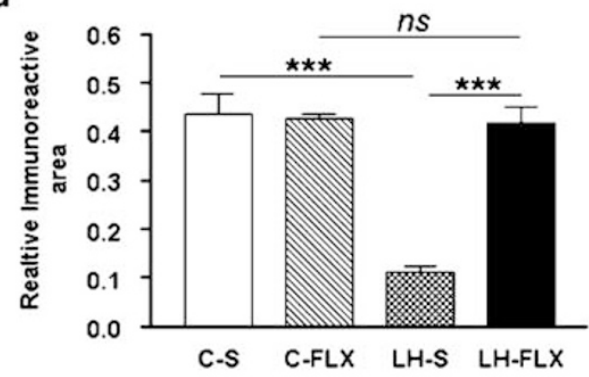

Figure 3 Fluoxetine (FLX) treatment induces synaptic remodeling in the hippocampus. Microphotographs show synaptophysin (SYN) immunostaining in the CA3 region of hippocampus from the experimental groups (a). Microphotographs show postsynaptic density 95 (PSD-95) immunostaining in the CA3 region of hippocampus from the experimental groups (b). Quantification of relative area of SYN immunoreactive structures revealed that 21 -day treatment with FLX of learned helplessness ( $\mathrm{LH}$ ) animals (LH-FLX group) reversed SYN labeling reduction induced by exposure to LH paradigm (c). Quantification of relative PSD-95 immunolabeled area demonstrated decreased immunostaining in the LH-S group that was fully reversed by FLX treatment (d). Data are expressed as mean values ( $\pm \mathrm{SD}$ ) of three immunohistochemical experiments; individual experiments consisted of three to five tissue sections of each of the five to six animals per group. ${ }^{*} * *<0.00 \mathrm{I}$; ns, nonsignificant between bars, two-way ANOVA followed by Bonferroni test. pyr, pyramidal cell layer, $\mathrm{SL}$, stratum lucidum; SO, stratum oriens; SR, stratum radiatum. Scale bar $=50 \mu \mathrm{m}$.

CA3 (Figure 4c), evidencing much less compact GAP-43 positive fiber bundles (Figure $4 c$, inset). FLX treatment of LH animals (LH-FLX) induced a slight increase of GAP-43 immunolabeled structures in both strata of CA3 (Figure 4d), specially affecting the bundle arrangement as shown by the highest degree of compaction when compared to LH-S animals (Figure $4 \mathrm{~d}$, inset).

\section{A Behaviorally Successful Treatment with FLX does not Reverse the Cytoskeletal Alterations Induced by Exposure to LH Paradigm}

Regarding the hippocampal cytoskeletal alterations, NFL was the only protein among the studied ones that was found diminished in the CA3 and DG regions of $\mathrm{LH}$ animals treated with saline for 21 days (LH-S) (Table 1). These results are in agreement with our previous report indicating that the hippocampal NFL reduction persists for at least 25 days after the LH induction (Reinés et al, 2004). Chronic treatment with FLX induced no effect on control animals (C-FLX group) in comparison with saline-injected ones (C-S group) and even though chronic FLX administration corrected the behavioral deficit, no effects were observed on NF subunits or MAP-2 either in the CA3 or in the DG as evidenced by the absence of statistical differences in the corresponding immunostainings between LH-FLX and LH-S groups (Table 1).

\section{FLX Withdrawal Induces the Recurrence of Despaired Behavior}

LH animals that received saline for 21 days and thereafter were kept in standard conditions for 90 days (LH-S/W) 
a
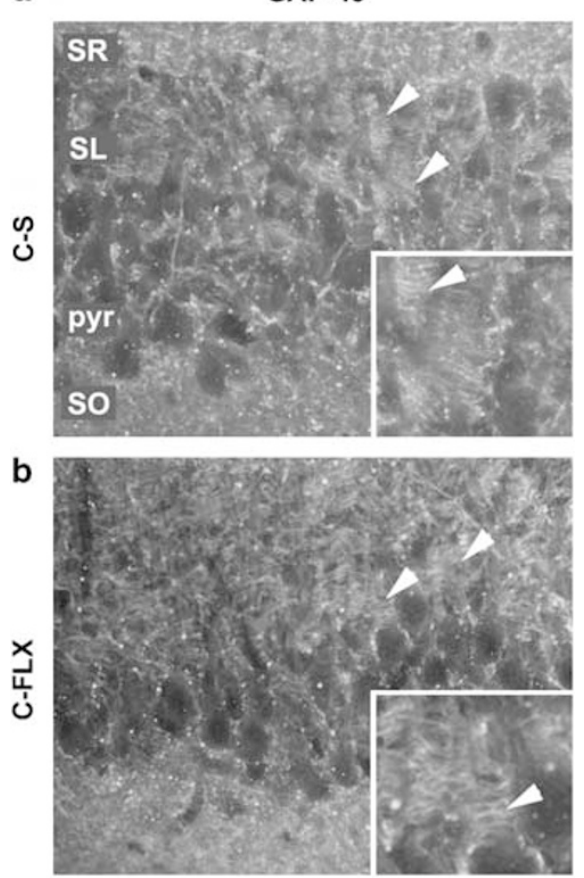

c

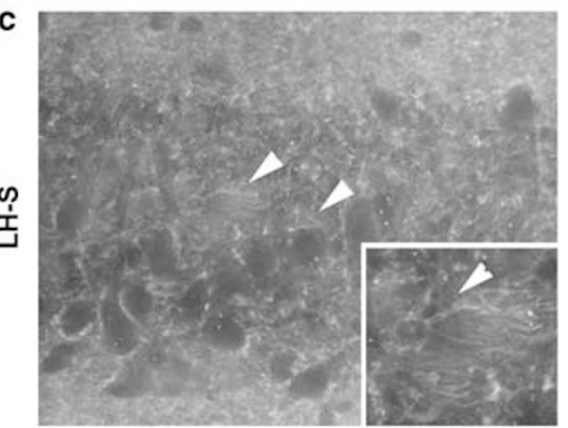

d

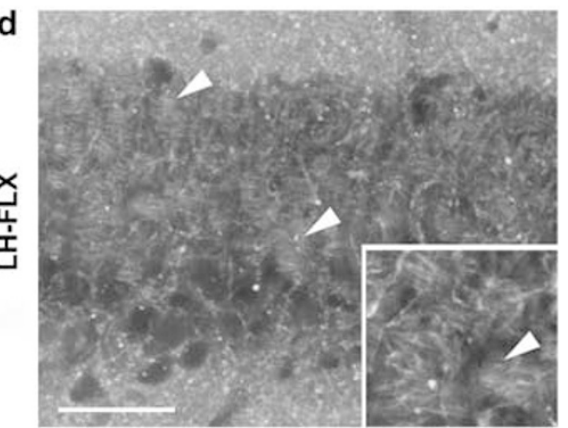

Figure 4 The axonal remodeling marker growth-associated protein 43 (GAP-43) is affected by exposure to learned helplessness (LH) paradigm as well as by fluoxetine (FLX) treatment. Photographs show GAP-43 positive structures in the CA3 region of the hippocampus. Intense immunolabeled fibers were observed in stratum oriens, lucidum, and radiatum of control animals ( $a$ and $b$ ). High magnification of GAP-43 fiber bundles ( $a$ and b, insets). Animals that developed despaired behavior and were treated for 21 days with saline (LH-S) showed lower GAP-43 immunostaining (c) evidencing lax fiber bundles (c, inset). FLX treatment of $L H$ animals (LH-FLX) increased GAP-43 positive structures (d) and fiber compaction when compared to LH-S animals (d, inset). pyr, pyramidal cell layer, $\mathrm{SL}$, stratum lucidum; SO, stratum oriens; SR, stratum radiatum. Arrow heads: GAP-43 fiber bundles. Scale bar $=50 \mu \mathrm{m}$.

exhibited increased escape latency, hallmark of the persistence of despaired behavior after 115 days $(P<0.05$ vs control animals, Kruskal-Wallis test followed by Dunn's multiple comparison test) (Figure 5). Since experiments carried out at day 25 showed no difference either in the escape latency or in the plastic markers of C-S and C-FLX animals, rats chronically treated with saline and kept in standard conditions for 90 days were employed as controls on day 115 (C). FLX withdrawal in LH-FLX animals that had responded to treatment on day 25 (LH-FLX/W group) induced the recurrence of the behavioral deficit as evidenced by the increment in the escape latency when tested on day $115(P<0.05 v s$ control, Kruskal-Wallis test followed by Dunn's multiple comparison test) (Figure 5).

\section{Reversion of FLX-Induced Synaptic Remodeling Accompanies the Recurrence of Despaired Behavior after Drug Withdrawal}

Persistence of despaired behavior in LH-S/W animals for 115 days was accompanied by diminished SYN and PSD-95 positive structures in the CA3 region of hippocampus (Figure $6 \mathrm{a}$ and $\mathrm{c}$, middle panels). Quantitative analysis of the immunolabeled structures showed 73 and $60 \%$ reductions for SYN $(P<0.001$ vs control, one-way ANOVA followed by Bonferroni test) and PSD-95 $(P<0.01$ vs control, one-way ANOVA followed by Bonferroni test), respectively (Figure $6 \mathrm{~b}$ and $\mathrm{d}$ ).

In addition to the recurrence of despaired behavior, the LH-FLX/W group displayed a significant decrement in SYN and PSD-95 immunostainings in CA3 (Figure 6a and c, bottom panels). Full reversion of the FLX effect found at day 25 on these two synaptic proteins was confirmed by quantitative analysis of the immunolabeled area. Reductions of 52 and $72 \%$ were obtained for SYN and PSD-95, respectively, in the LH-FLX/W group $(P>0.05$ vs LH-S/W animals (Figure $6 \mathrm{~b}$ and $\mathrm{d}$ ).

On the contrary, quantification of SYN and PSD-95 positive structures in the DG of LH-S/W on day 115 showed, in coincidence with that was found at day 25, that persistence of despaired behavior was not related to changes in the immunolabelings for these two proteins in this hippocampal area $(P>0.05$ vs control, one-way ANOVA). Consequently to the lack of FLX effect on these synaptic markers in the DG of LH animals on day 25, interruption of the pharmacological treatment for 90 days was not accompanied by changes in SYN or PSD-95 markers in the LH-FLX/W group $(P>0.05$ vs LH-S/W, one-way ANOVA). Relative immunolabeled areas in the DG were as follows: for SYN, $0.331 \pm 0.014$ in $\mathrm{C}$; $0.317 \pm 0.011$ in $\mathrm{LH}-$ $\mathrm{S} / \mathrm{W}$, and $0.337 \pm 0.010$ in LH-FLX/W group. For PSD-95, $0.187 \pm 0.012$ in $\mathrm{C} ; 0.183 \pm 0.010$ in $\mathrm{LH}-\mathrm{S} / \mathrm{W}$, and $0.192 \pm 0.013$ in LH-FLX/W group.

Different results were obtained for GAP-43 in the CA3 region as shown in Figure 6e for control, LH-S/W and LHFLX/W groups at 115 days. Control animals evidenced the same immunostaining pattern as described at 25 days in Figure $4 \mathrm{a}$, in which GAP-43 positive fibers were predominant in stratum lucidum and radiatum and arranged in dense bundles (Figure 6e, top panel). LH-S/W group revealed a persistent decrease of GAP-43 immunostaining accompanied by lax bundles of fibers in stratum lucidum. Interestingly, the effect of 21-day treatment with FLX (LHFLX/W) remained after the 90-day withdrawal period as shown by the higher GAP-43 immunostaining compared to 
Table I Immunoreactive Area $\left(\mu \mathrm{m}^{2}\right)$ of NFL, NFM, NFH, and MAP-2 Fibers in the CA3 and DG Areas of the Hippocampus

\begin{tabular}{|c|c|c|c|c|c|c|c|c|}
\hline \multirow{2}{*}{$\begin{array}{l}\text { Experimental } \\
\text { groups }\end{array}$} & \multicolumn{2}{|c|}{ NFL } & \multicolumn{2}{|c|}{ NFM } & \multicolumn{2}{|c|}{ NFH } & \multicolumn{2}{|c|}{ MAP-2 } \\
\hline & CA3 & DG & CA3 & DG & CA3 & DG & CA3 & DG \\
\hline C-S & $3621 \pm 589$ & $4678 \pm 609$ & $4791 \pm 714$ & $5727 \pm 737$ & $5593 \pm 891$ & $6860 \pm 528$ & $4956 \pm 509$ & $5005 \pm 217$ \\
\hline C-FLX & $2895 \pm 108$ & $4050 \pm 610$ & $4291 \pm 920$ & $5198 \pm 1293$ & $5704 \pm 345$ & $6619 \pm 1189$ & $4310 \pm 297$ & $4635 \pm 393$ \\
\hline LH-S & $874 \pm 83^{* * * *}$ & $1024 \pm 111 *$ & $4237 \pm 580$ & $5409 \pm 1093$ & $5224 \pm 570$ & $6582 \pm 737$ & $5302 \pm 886$ & $5430 \pm 806$ \\
\hline
\end{tabular}

Abbreviations: DG, dentate gyrus; MAP-2, microtubule-associated protein 2; NFL, light neurofilament subunit; NFH, heavy neurofilament subunit.

Immunoreactive area in $\mu^{2}$ of NFL, NFM, NFH, and MAP-2 fibers in the CA3 and hilar region of the DG of hippocampus from controls treated for 21 days with saline (C-S) or fluoxetine (C-FLX) and learned helplessness group treated for 21 days with saline (LH-S) or fluoxetine (LH-FLX). Data are expressed as mean values ( \pm SD) of four immunohistochemical experiments; individual experiments consisted of three to five tissue sections of each of the five to six animals per group. * $P<0.05$ and **** $P<0.00$ I vs corresponding control by two way-ANOVA followed by Bonferroni test.

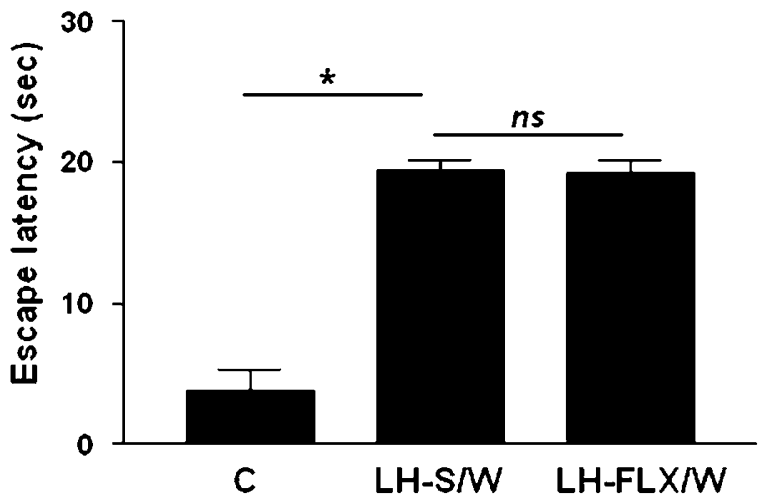

Figure 5 Recurrence of depressive-like behavior after fluoxetine ( $\mathrm{L} L$ ) withdrawal. Learned helplessness $(\mathrm{LH})$ animals treated for 21 days with FLX exhibited recurrence of despaired behavior after the 90-day withdrawal period of pharmacological treatment (LH-FLXN group) as shown by the absence of statistical difference in the escape latency (sec) when compared to $\mathrm{LH}$ animals treated for 21 days with saline and kept in the same conditions for 90 days (LH-S/W group). Data are expressed as mean values $( \pm S D, n=5-6)$. $* P<0.05$; ns, nonsignificant between bars, Kruskal-Wallis test.

LH-S/W group and by the control-like bundle structures found in stratum lucidum of CA3 region (Figure 6e middle and bottom panels).

\section{Hippocampal NFL Diminution is a Common Trait in Long-Lasting Despaired Behavior and Recurrence of Behavioral Deficit}

The long-lasting despaired behavior disclosed in LH-S/W animals on day 115 was as well accompanied by a persistent reduction in NFL immunostaining in the CA3 region $(P<0.001$ vs control, one-way ANOVA followed by Bonferroni test) (Figure $7 \mathrm{a}$ and b). Similarly, and in accordance with the above-mentioned findings showing that FLX treatment failed to reverse NFL reduction on day 25, NFL immunostaining was found decreased in the CA3 region of LH-FLX/W group on day $115(P<0.001 v s$ control, one-way ANOVA followed by Bonferroni test) (Figure $7 \mathrm{a}$ and $\mathrm{b}$ ).

LH-S/W animals also showed a persistent diminution of NFL immunostaining in the DG region of hippocampus
$(P<0.001$ vs control, one-way ANOVA followed by Bonferroni test). As expected by the lack of FLX effect in the DG on day 25, NFL immunostaining was found as well decreased in this hippocampal area of LH-FLX/W group on day $115(P<0.001 v s$ control, one-way ANOVA followed by Bonferroni test). NFL immunoreactive areas $\left(\mu \mathrm{m}^{2}\right)$ in the DG were as follows: $4126 \pm 566$ for control, $830 \pm 407$ for LH-S/W, and $1141 \pm 80$ for LH-FLX/W.

\section{DISCUSSION}

In the present work, we show that when LH animals are chronically treated with FLX for 21 days, as expected from other reports (Dulawa et al, 2004), reversion of depressivelike behavior is observed. We demonstrate that a behaviorally successful treatment with the antidepressant FLX fully reverses the diminution of synaptic proteins induced in the CA3 by exposure to the LH paradigm, an animal model of depression. Axonal remodeling is also triggered as another consequence of the pharmacological treatment. When the antidepressant is withdrawn, the behavioral deficit recurs and the diminution of synaptic proteins is again manifested. Interestingly, FLX effect on GAP-43 seems to persist after interrupting the drug treatment. The fact that synaptic proteins were found unchanged in the DG of LH animals suggests the regional specificity for the stress-induced synaptic changes and for the FLX-dependent behavioral improvement. With regards to neuronal cytoskeleton, chronic treatment with FLX has no effect on the different proteins studied. Since NFL diminution observed in hippocampal CA3 region of $\mathrm{LH}$ animals has proved to be a long-lasting effect, this structural alteration has been concomitantly found in animals that showed recurrence of despaired behavior after FLX withdrawal.

It is worth noticing that despite animal models share some features with the clinical picture observed in depressed human beings (i.e. changes in hippocampal connectivity and antidepressant response), prominent differences are inherent to the different evolutionary stages of both species (subjective suffering, guilt and ruin thoughts, death, or suicide ideas) (Markowitz and Milrod, 2005), therefore limiting the potential extrapolation of preclinical results to the clinical setting. 
a
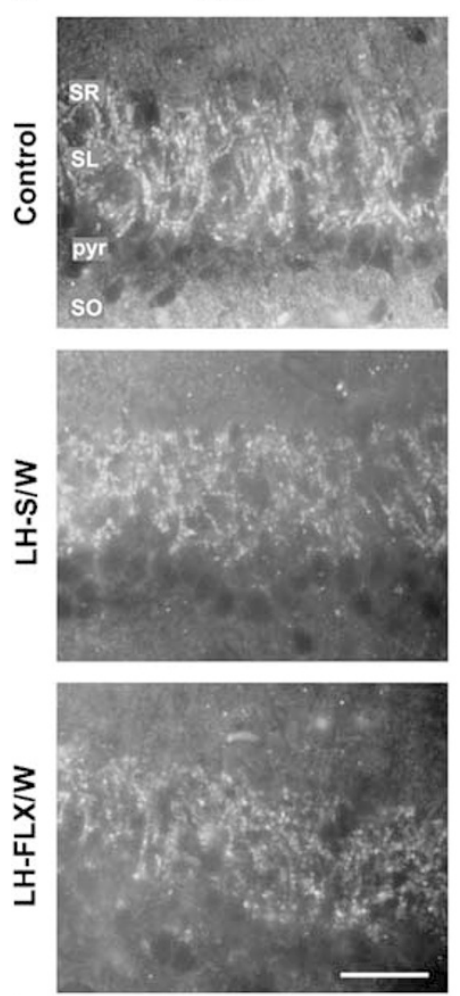

b

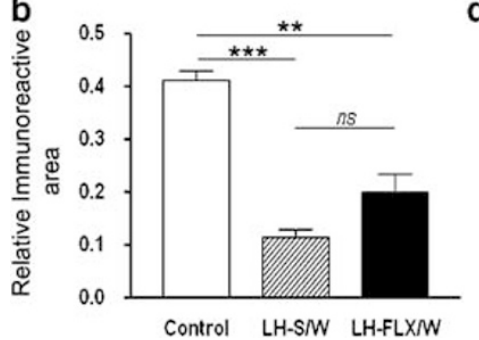

c
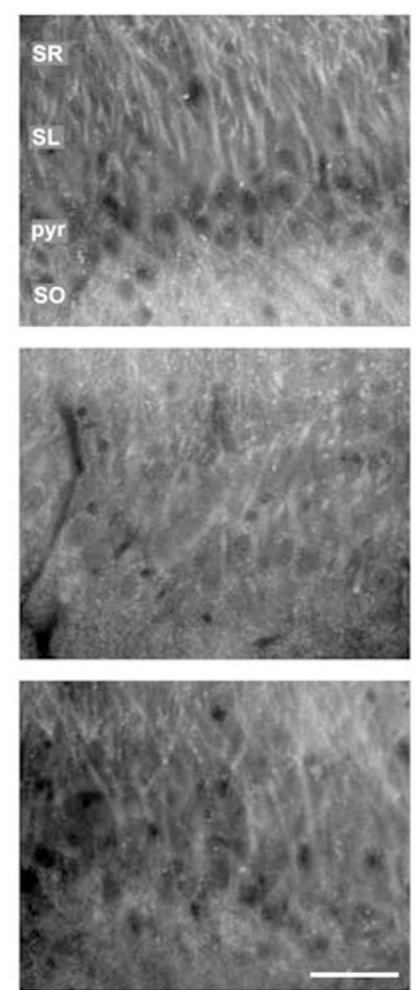

d

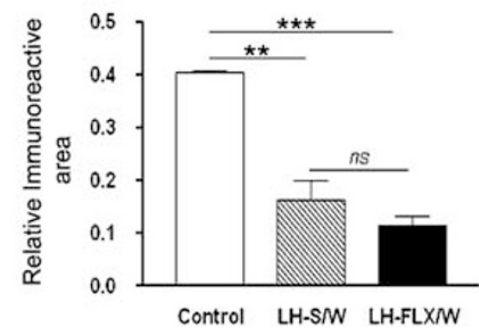

e
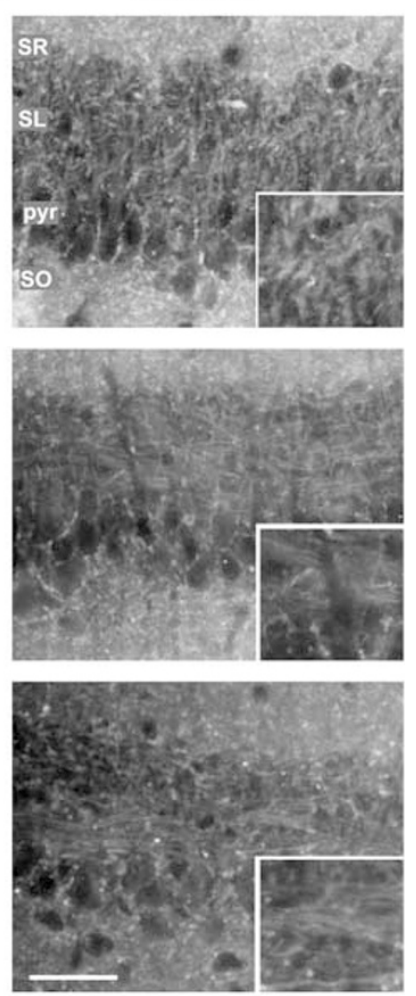

GAP-43

Figure 6 Fluoxetine (FLX) withdrawal reverses the hippocampal remodeling induced by administration of the drug. Microphotographs show synaptophysin (SYN) (a) and postsynaptic density 95 (PSD-95) (c) immunostainings in the CA3 region of hippocampus from the experimental groups at day I 15. Quantitative analysis of relative area of immunolabeled structures revealed persistent diminution in LH-S/W animals of SYN (b) and PSD-95 (d) and reversion of the FLX effect observed at day 25 after FLX withdrawal (LH-FLX/W). LH-S/W also showed lower growth-associated protein 43 (GAP-43) immunostaining accompanied by lax bundles of fibers in stratum lucidum and radiatum. The effect of $2 \mathrm{I}$-day treatment with FLX (LH-FLX/W) remained after the 90-day withdrawal period as shown by the higher GAP-43 immunostaining compared to LH-S/W group (e). Data are expressed as mean values $( \pm S D)$ of three immunohistochemical experiments; individual experiments consisted of three to five tissue sections of each of the five to six animals per group. *** $<0.0$ I; **** $P<0.00$ I; ns, nonsignificant between bars, one-way ANOVA followed by Bonferroni test. pyr, pyramidal cell layer; $S$, stratum lucidum; $\mathrm{SO}$, stratum oriens; SR, stratum radiatum. Scale bar $=50 \mu \mathrm{m}$.

\section{Modulation of Synaptic Plasticity by Antidepressants}

When studying synaptic proteins in the CA3 region of the hippocampus, we found that the $\mathrm{LH}$ paradigm induces a large decrease of SYN and PSD-95, pre- and postsynaptic markers, respectively. We also observed that axonal remodeling is reduced in LH animals as indicated by the decrement in GAP43. The fact that synaptic proteins were found unchanged in the DG of LH animals suggests the regional specificity for the stress-induced synaptic changes. These results are in line with those reported by Thome et al (2001) and Kuroda and McEwen (1998) showing, respectively, diminished SYN and GAP-43 mRNA levels in stressed rats and support the hypothesis that brain plasticity might be disturbed in experimental models of depression (Duman, 2002).
Changes in the strength of synaptic transmission as observed in the long-term depression induced by stress in rats (Holderbach et al, 2006) or in the long-term potentiation (LTP) reported as a consequence of rat antidepressant treatment (Holderbach et al, 2006) are considered related to modifications of synaptic components. For instance, it is known that late phases of LTP induced in rat organotypic culture involve gene transcription, protein synthesis, and ultimately synaptogenesis (Toni et al, 1999; Engert and Bonhoeffer, 1999) that finally lead to permanent changes in the neuronal network. Under our conditions, FLX treatment induced increments in the immunostainings for pre- and postsynaptic markers in the CA3 hippocampal region. It is known that SYN is the major integral protein of synaptic vesicles and reduced SYN is consistent with a decrease in 


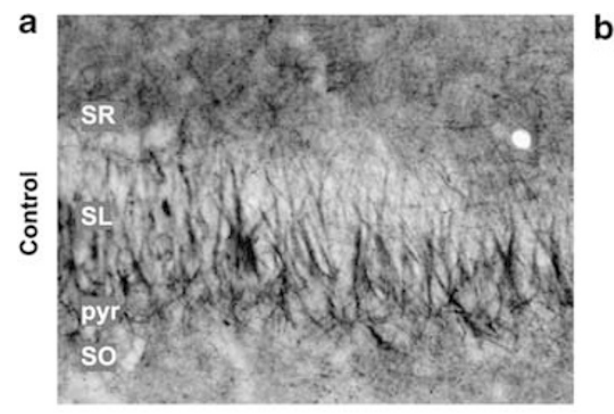

\section{b}
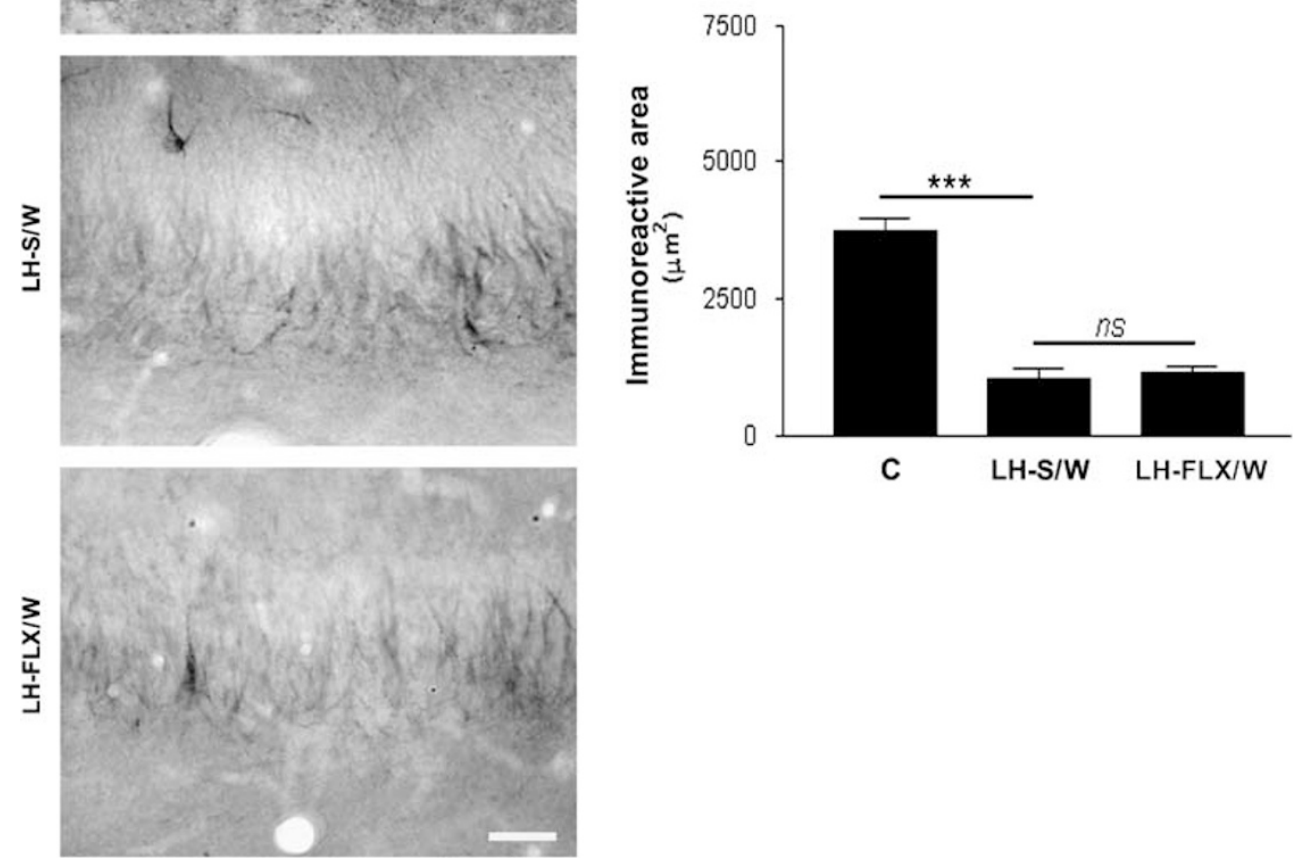

Figure 7 Persistence of NLF diminution after fluoxetine $(F L X)$ withdrawal. Bright field photographs show NFL immunolabeled structures of the CA3 region of hippocampus from the experimental groups at day II5 (a). Quantification of immunoreactive area of NFL fibers disclosed a persistent NFL decrease in LH-S/W animals as well as in those in which the depressive-like behavior recurs after FLX withdrawal (b). Data are expressed as mean values $( \pm S D$ ) of three immunohistochemical experiments; individual experiments consisted of three to five tissue sections of each of the five to six animals per group. *** $P<0.00 \mathrm{I}$; ns, nonsignificant between bars, one-way ANOVA followed by Bonferroni test. pyr, pyramidal cell layer; SL, stratum lucidum; $\mathrm{SO}$, stratum oriens; SR, stratum radiatum. Scale bar $=40 \mu \mathrm{m}$.

synaptic density (Eastwood and Harrison, 2001). Therefore, return to normal SYN levels under FLX treatment might suggest the formation of new synaptic contacts in the CA3 region of the hippocampus. Taking into account that the analyzed changes have a specific localization within the stratum lucidum, area of glutamatergic mossy fiber arrival, reversion to normal levels of PSD-95 (an exclusive postsynaptic protein of excitatory synapses) after FLX treatment, further support this contention. By doing so, it is likely that FLX may improve synaptic neurotransmission and therefore, overcomes the behavioral deficit observed in LH animals. SYN changes induced by FLX may also reflect a different functional status of CA3 synapses since increased levels of synaptic vesicle proteins have been described to accompany in vivo induction of LTP in rats (Lynch et al, 1994; Mullany and Lynch, 1997) and increments of SYN immunoreactivity have been shown in kindling (Li et al, 2002).

It is very tempting to speculate that the increased GAP-43, we observed under FLX administration could contribute to the final effect on synapse remodeling since this protein has been described to play important roles in the regulation of cytoskeletal organization in the nerve ending of cultured cells as well as in the formation of new synapses and in the enhanced axonal sprouting observed after brain injury in adult mice (Benowitz and Routtenberg, 1997).

A possible candidate to be responsible for stress- and antidepressant-induced modulation of synaptic plasticity is the brain-derived neurotrophic factor (BDNF). This factor has been found diminished in the rat hippocampus after acute and chronic stress (Smith et al, 1995; Scaccianoce et al, 2003) and has proved to possess antidepressant action in different models of depression (Shirayama et al, 2002). Moreover, chronic treatment with FLX promotes BDNF expression in the rat hippocampus (De Foubert et al, 2004) that in turn leads to activation of genes and biochemical pathways involved in plasticity and survival (Mattson et al, 2004). At synaptic structural level, adult BDNF knockout mice show reduced number of vesicles docked at presynaptic active zones and marked decreased levels of SYN and synaptobrevin (Pozzo-Miller et al, 1999), alterations that can be reversed by BDNF administration (Pozzo-Miller et al, 1999), and somewhat resemble the synaptic protein decrease we observed in LH animals. On the other hand, 
and similarly to the SYN and PSD-95 increments we found after FLX administration to LH animals, BDNF treatment of wild-type rat organotypic cultures increments synaptic density and the size of the docked synaptic vesicles pool (Tyler and Pozzo-Miller, 2001) while producing greater levels of synaptic proteins (Tartaglia et al, 2001). Inhibition of BDNF expression in FLX-treated rats by means of oligonucleotide antisense administration could provide valuable information about the actual role of this neurotrophin in synaptic remodeling.

\section{Synaptic Plasticity and Recurrence of Despaired Behavior after Antidepressant Withdrawal}

In agreement with the clinical observations, we showed that despaired behavior reappears after interrupting the pharmacological treatment. Drug withdrawal also reverses the synaptic plastic changes observed in the CA3 hippocampal region under FLX administration with the only exception of GAP-43. It is worth noticing that the most affected proteins were those directly involved in synapse morphology and function as SYN and PSD-95. Not only the response of CA3 synaptic proteins to FLX administration and withdrawal, but also the lack of changes in the DG of LH animals after its administration strengthens the notion that a regional specificity might be involved in the FLX action. Our results also suggest that even though the synaptic changes induced by antidepressants may be able to correct the behavioral deficit, those changes tend to be labile and seem to require for their maintenance a continuous or at least prolonged exposure to the drug. Since higher FLX doses have also proved to be effective in improving the behavioral deficit (Zazpe et al, 2007), it might be interesting to investigate whether higher FLX doses induce more persistent effects upon withdrawal.

Synaptic stabilization is a crucial mechanism that has to be perfectly balanced with synaptic plasticity to support the normal function of the neuronal network in the adult brain (Schulz, 2006). At this level, experimental evidences point out once again to BDNF as an appealing candidate. Besides its role in synaptogenesis (Tyler and Pozzo-Miller, 2001; Hu et $a l, 2005)$ and synaptic ultra structural composition (Pozzo-Miller et al, 1999; Tartaglia et al, 2001; Tyler and Pozzo-Miller, 2001; Carter et al, 2002) in developing and adult brain, this neurotrophin promotes synaptic connectivity by modulating maturation of developing synapses in culture (Collin et al, 2001) and by stabilizing in vivo synapses already established ( $\mathrm{Hu}$ et al, 2005). Taken into account that BDNF has been proposed to mediate FLX action, it can be hypothesized that antidepressant withdrawal might drop down BDNF levels to the abnormally low concentrations described in stressed animals leading to the reversion of synaptic changes we observed under FLX treatment. In fact, it has been shown that BDNF effects on synapse maturation and stabilization do not longer persist after discontinuation of BDNF treatment (Collin et al, 2001).

\section{Modulation of Cytoskeletal Plasticity by Antidepressants}

As indicated by the persistence of NFL diminution and by the absence of changes in the other NF subunits or MAP-2, we show that FLX action seems not to involve cytoskeletal plastic changes. Similar observations were made despite of increasing the length of treatment to 42 days (data not shown). In accordance with our results, Magariños et al (1999) have previously reported that while FLX fails to reverse the rat hippocampal dendritic atrophy as measured by Golgi staining, tianeptine proved to be effective. Therefore, our findings further support the notion that correction of cytoskeletal alterations is not a common action among antidepressants (Magariños et al, 1999).

In addition, MRI measurement of hippocampal volume of patients suffering from depression could not consistently detect any significant change after successful antidepressant treatment (Videbech and Ravnkilde, 2004). Furthermore, spontaneous reversion of the affective disorder is not paralleled by a normalization of the hippocampal volume when compared to control subjects (Neumeister et al, 2005). Interestingly, clinical experience and follow-up studies of depressive patients successfully treated with antidepressants have demonstrated that over $50 \%$ of them suffer from recurrence of the disease months or years after drug withdrawal (Shelton, 2004). Since NFL diminution is a common trait between persistent despaired behavior and its recurrence after FLX withdrawal, it may be speculated based on our findings that this cytoskeletal alteration might contribute to the labile effect of FLX on synapses. In fact, it has been proposed a role for NFL in scaffolding synaptic components as this NF subunit is linked to postsynaptic density proteins (Hirao et al, 2000; Terry-Lorenzo et al, 2000)

All these results seem to be, at least in part, in contradiction with those reported by Magariños et al (1999) who observed that tianeptine, an atypical antidepressant, but not FLX prevents the shrinkage of apical dendrites in CA3 pyramidal neurons of stressed rats. Although, intuitively, dendrite shrinkage may be correlated with a decrement in synaptic markers, this association remains to be proved. Indeed, an increment in the synapse number or even functional changes of the synapses on the remaining dendritic projections might also be plausible. Moreover, Magariños et al searched for the prevention of the dendrite atrophy, an approach rather different from ours, since we were looking for the reversion of the decrement of synaptic markers already present. To further understand these apparently conflicting results, the potential relationships between dendrite morphology on one hand and SYN and PSD-95 staining on the other should be further investigated.

To summarize, our results indicate that synaptic plasticity is a pharmacological target strongly involved in the behavioral efficacy of FLX. Synaptic remodeling could underlie the behavioral correction despite the absence of cytoskeletal remodeling. Surprisingly, reversion of the synaptic changes induced by FLX administration is accompanied by recurrence of despaired behavior 3 months after the interruption of the treatment. These results mimic a clinically meaningful phenomenon since one of the most compelling limitations of the antidepressant treatment today is the necessity of continuous administration to provide permanent clinical benefits (Nemeroff, 2007). The results presented in this paper suggest that mechanisms involved in the stabilization of synaptic changes may 
become interesting targets to be investigated with regard to the treatment of depression and its recurrence.

\section{ABBREVIATIONS}

BDNF, brain-derived neurotrophic factor; CA3, CA3 region of Ammon's horn; DG, dentate gyrus; FLX, fluoxetine; GAP43, growth-associated protein 43; LH, learned helplessness; MAP-2, microtubule-associated protein 2; mf, mossy fibers; MRI, magnetic resonance imaging; NF, neurofilament; NFH, heavy neurofilament subunit; NFL, light neurofilament subunit; NFM, medium neurofilament subunit; PBS, phosphate-buffered saline; PSD-95, postsynaptic density 95; pyr, pyramidal cell layer; SL, stratum lucidum; SO, stratum oriens; SR, stratum radiatum; SYN, synaptophysin.

\section{ACKNOWLEDGEMENTS}

SW and AR are Investigators from Consejo Nacional de Investigaciones Científicas y Técnicas (CONICET). This work was supported by grants from Universidad de Buenos Aires (UBACYT M-013), Consejo Nacional de Investigaciones Científicas y Técnicas (PIP5870), and Agencia Nacional de Promoción Científica y Técnica (PICT 0511102, PICT 31953 and PICT 34397), Argentina.

\section{DISCLOSURE/CONFLICT OF INTEREST}

The author(s) declare that, except for income received from my primary employer, no financial support or compensation has been received from any individual or corporate entity over the past 3 years for research or professional service and there are no personal financial holdings that could be perceived as constituting a potential conflict of interest. No author has conflict of interests related to the present research.

\section{REFERENCES}

Akhondzadeh S (1999). Hippocampal synaptic plasticity and cognition. J Clin Pharm Ther 24: 241-248.

Anisman H, Merali Z (2001). Rodent models of depression: learned helplessness induced in mice. Curr Protoc Neurosci, Unit 8.10C.

Benowitz LI, Routtenberg A (1997). GAP-43: an intrinsic determinant of neuronal development and plasticity. Trends Neurosci 20: 84-91.

Bremner JD, Narayan M, Anderson ER, Staib LH, Miller HL, Charney DS (2000). Hippocampal volume reduction in major depression. Am J Psychiatry 157: 115-118.

Carter AR, Chen C, Schwartz PM, Segal RA (2002). Brain-derived neurotrophic factor modulates cerebellar plasticity and synaptic ultrastructure. J Neurosci 22: 1316-1327.

Chen B, Dowlatshahi D, MacQueen GM, Wang JF, Young LT (2001). Increased hippocampal BDNF immunoreactivity in subjects treated with antidepressant medication. Biol Psychiatry 15: $260-265$.

Cho OK, Hunt CA, Kennedy MB (1992). The rat brain postsynaptic density fraction contains a homolog of the drosophila dics-large tumor suppressor protein. Neuron 9: 929-942.

Collin C, Vicario-Abejon C, Rubio ME, Wenthold RJ, McKay RD, Segal M (2001). Neurotrophins act at presynaptic terminals to activate synapses among cultured hippocampal neurons. Eur $J$ Neurosci 13: 1273-1282.
Czéh B, Lucassen PJ (2007). What causes the hippocampal volume decrease in depression? Are neurogenesis, glial changes and apoptosis implicated? Eur Arch Psychiatry Clin Neurosci 257: 250-260.

Czéh B, Michaelis T, Watanabe T, Frahm J, de Biurrun G, van Kamper $M$ et al (2001). Stress-induced changes in cerebral metabolites, hippocampal volume and cell proliferation are prevented by antidepressant treatment with tianeptine. Proc Natt Acad Sci USA 98: 12796-12801.

De Foubert G, Carney SL, Robinson CS, Destexhe EJ, Tomlinson R, Hicks CA et al (2004). Fluoxetine-induced change in rat brain expression of brain-derived neurotrophic factor varies depending on length of treatment. Neuroscience 128: 597-604.

Donohue HS, Gabbott LA, Davies HS, Rodriguez JJ, Cordero MI, Sandi C et al (2006). Chronic restraint stress induces changes in synapse morphology in stratum lacunosum-moleculare CA1 rat hippocampus: a stereological and three-dimensional ultrastructural study. Neuroscience 140: 597-606.

Dulawa SC, Holick KA, Gundersen B, Hen R (2004). Effects of chronic fluoxetine in animal models of anxiety and depression. Neuropsychopharmacology 29: 1321-1330.

Duman RS (2002). Pathophysiology of depression: the concept of synaptic plasticity. Eur Psychiatry 17: 306-310.

Eastwood SL, Harrison PJ (2001). Synaptic pathology in the anterior cingulated cortex in schizophrenia and mood disorders. A review and western blot study of synaptophysin, GAP-43 and the complexins. Brain Res Bull 55: 569-578.

Eichenbaum H, Otto T (1992). The hippocampus - what does it do? Behav Neural Biol 57: 2-36.

Engert F, Bonhoeffer T (1999). Dendritic spine changes associated with hippocampal long-term synaptic plasticity. Nature 399: 66-70.

Ferrero AJ, Cereseto M, Sifonios LL, Reines A, Peixoto E, Rubio MC et al (2007). Cytoskeleton of hippocampal neurons as a target for valproic acid in an experimental model of depression. Prog Neuropsychopharmacol Biol Psychiatry 31: 1419-1428.

Grillo CA, Piroli GG, Wood GE, Reznikov LR, McEwen BS, Reagan LP (2005). Immunocytochemical analysis of synaptic proteins provides new insights into diabetes-mediated plasticity in the rat hippocampus. Neuroscience 136: 477-486.

Hardman JG, Limbird LE (2001). Goodman and Gilman, The Pharmacological Basis of Therapeutics, 10th edn. McGraw-Hill Co: Atlampa, Mexico.

Hirao K, Hata Y, Deguchi M, Yao I, Ogura M, Rokukawa C et al (2000). Association of synapse-associated protein 90/postsynaptic density-95-associated protein (SAPAP) with neurofilaments. Genes Cells 5: 203-210.

Holderbach R, Clark K, Moreau J-L, Bischofberger J, Normann C (2006). Enhanced long-term synaptic depression in an animal model of depression. Biol Psychiat 62: 92-100.

$\mathrm{Hu}$ B, Nikolakopoulou AM, Cohen-Cory S (2005). BDNF stabilizes synapses and maintains the structural complexity of optic axons in vivo. Development 132: 4285-4298.

Hunt CA, Schenker LJ, Kennedy MB (1996). PSD-95 is associated with the postsynaptic density and not with the presynaptic membrane at forebrain synapses. J Neurosci 16: 1380-1388.

Iwata M, Shirayama Y, Ishida H, Kawahara R (2006). Hippocampal synapsin I, growth-associated protein-43, and microtubuleassociated protein-2 immunoreactivity in learned helplessness rats and antidepressant-treated rats. Neuroscience 141: $1301-1313$.

Keller MB (2001). Long-term treatment of recurrent and chronic depression. J Clin Psychiatry 62: 3-5.

Kuroda Y, McEwen BS (1998). Effect of chronic restraint stress and tianeptine on growth factors, growth-associated protein-43 and microtubule-associated protein 2 mRNA expression in the rat hippocampus. Brain Res Mol Brain Res 59: 35-39. 
Li S, Reinprecht I, Fahnestock M, Racine RJ (2002). Activitydependent changes in synaptophysin immunoreactivity in hippocampus, piriform cortex, and entorhinal cortex of the rat. Neuroscience 115: 1221-1229.

Lynch MA, Voss KL, Rodriguez J, Bliss TV (1994). Increase in synaptic vesicle proteins accompanies long-term potentiation in the dentate gyrus. Neuroscience 60: 1-5.

Magariños AM, Deslandes A, McEwen BS (1999). Effects of antidepressant and benzodiazepine treatments on the dendritic structure of CA3 pyramidal neurons after chronic stress. Eur J Pharmacol 371: 113-122.

Magariños AM, McEwen BS, Flugge G, Fuchs E (1996). Chronic psychosocial stress causes apical dendritic atrophy of hippocampal CA3 pyramidal neurons in subordinate tree shrews. J Neurosci 16: 3534-3540.

Malberg JE, Eisch AJ, Nestler EJ, Duman RS (2000). Chronic antidepressant treatment increases neurogenesis in adult rat hippocampus. J Neurosci 20: 9104-9110.

Markowitz JC, Milrod B (2005). Mood disorders: intrapsychic and interpersonal aspects. In: Sadock BJ, Sadock VA (eds). Comprehensive Textbook of Psychiatry. Lippincott Williams \& Wilkins: Philadelphia, pp 1603-1611.

Mattson MP, Maudsley S, Martin B (2004). BDNF and 5-HT: a dynamic duo in age-related neuronal plasticity and neurodegenerative disorders. Trends Neurosci 27: 589-594.

Mervaala E, Fohr J, Kononen M, Valkonen-Korhonen M, Vainio P, Partanen K et al (2002). Quantitative MRI of the hippocampus and amygdala in severe depression. Psychopharmacol Med 30: $117-125$.

Mullany P, Lynch MA (1997). Changes in protein synthesis and synthesis of the synaptic vesicle protein, synaptophysin, in enthorinal cortex following induction of long-term potentiation in dentate gyrus: an age-related study in the rat. Neuropharmacology 36: 973-980.

Nakagawa Y, Sasaki A, Takashima T (1999). The Gaba ${ }_{B}$ receptor antagonist CGP36742 improves learned helplessness in rats. Eur J Pharmacol 381: 1-7.

Nemeroff CB (2007). Prevalence and management of treatmentresistant depression. J Clin Psychiatry 68: 17-25.

Neumeister A, Wood S, Bonne O, Nugent AC, Luckenbaugh DA, Young $\mathrm{T}$ et al (2005). Reduced hippocampal volume in unmedicated, remitted patients with major depression versus control subjects. Biol Psychiatry 57: 935-937.

Nibuya M, Morinobu S, Duman RS (1995). Regulation of BDNF and trkB mRNA in rat brain by chronic electroconvulsive seizure and antidepressant drug treatments. J Neurosci 15: 7539-7547.

Nibuya M, Nestler EJ, Duman RS (1996). Chronic antidepressant administration increases the expression of cAMP response element binding protein (CREB) in rat hippocampus. $J$ Neurosci 16: 2365-2372.

Paxinos G, Watson C (1986). The Rat Brain in Stereotaxis Coordinates, 4th edn. Academic Press: San Diego, CA.

Posternak MA, Zimmerman M (2007). Therapeutic effect of followup assessments on antidepressant and placebo response rates in antidepressant efficacy trials: meta-analysis. Br J Psychiatry 190: 287-292.

Pozzo-Miller LD, Gottschalk W, Zhang L, McDermott K, Du J, Gopalakrishnan R et al (1999). Impairments in high-frequency transmission, synaptic vesicle docking, and synaptic protein distribution in the hippocampus of BDNF knockout mice. J Neurosci 19: 4972-4983.

Ramos AJ, Tagliaferro P, Lopez EM, Pecci Saavedra JP (2000). Neuroglial interactions in a model of para-clorphenylalanineinduced serotonin depletion. Brain Res 883: 1-14.

Reinés A, Cereseto M, Ferrero A, Bonavita C, Wikinski S (2004). Neuronal cytoskeletal alterations in an experimental model of depression. Neuroscience 129: 529-538.
Rosenbrock H, Koros E, Bloching A, Podhorna J, Borsini F (2005). Effect of chronic intermittent restraint stress on hippocampal expression of markers for synaptic plasticity and progenitor cell proliferation in rats. Brain Res 1040: 55-63.

Scaccianoce S, Del Bianco P, Caricasole A, Nicoletti F, Catalani A (2003). Relationship between learning, stress, and hippocampal brain-derived neurotrophic factor. Neuroscience 121: 825-828.

Schulz DJ (2006). Plasticity and stability in neuronal output via changes in intrinsic excitability: it's what's inside that counts. $J$ Exp Biol 209: 4821-4827.

Sheline Y, Sanghavi M, Mintun MA, Gado MH (2000). MRI studies of neuroanatomic changes in unipolar major depression: the role of stress and medical comorbidity. Biol Psychiatry 48: 791-800.

Shelton CI (2004). Long-term management of major depressive disorder: are differences among antidepressant treatments meaningful? J Clin Psychiatry 65: 29-33.

Shirayama Y, Chen AC, Nakagawa S, Russell DS, Duman RS (2002). Brain-derived neurotrophic factor produces antidepressant effects in behavioural models of depression. J Neurosci 22: 3251-3261.

Smith MA, Makino S, Kvetnansky R, Post RM (1995). Stress and glucocorticoids affect the expression of brain-derived neurotrophic factor and neurotrophin-3 mRNAs in the hippocampus. J Neurosci 15: 1768-1777.

Tagliaferro P, Vega MD, Evrard SG, Ramos AJ, Brusco A (2002). Alcohol exposure during adulthood induces neuronal and astroglial alterations in the hippocampal CA-1 area. Ann NY Acad Sci 965: 334-363.

Tartaglia N, Du J, Tyler WJ, Neale E, Pozzo-Miller L, Lu B (2001). Protein synthesis-dependent and -independent regulation of hippocampal synapses by brain-derived neurotrophic factor. J Biol Chem 276: 37585-37593.

Terry-Lorenzo RT, Inoue M, Connor JH, Haystead TAJ, Armbruster BN, Gupta RP et al (2000). Neurofilament-L is a protein phosphatase-1-binding protein associated with neuronal plasma membrane and post-synaptic density. J Biol Chem 275: 2439-2446.

Thome J, Pesold B, Baader M, Hu M, Gewirtz JC, Duman RS et al (2001). Stress differentially regulates synaptophysin and synaptotagmin expression in hippocampus. Biol Psychiatry 50: 809-812.

Toni N, Buchs PA, Nikonenko I, Bron CR, Muller D (1999). LTP promotes formation of multiple spine synapses between a single axon terminal and a dendrite. Nature 402: 421-425.

Tyler WJ, Pozzo-Miller LD (2001). BDNF enhances quantal neurotransmitter release and increases the number of docked vesicles at the active zones of hippocampal excitatory synapses. J Neurosci 21: 4249-4258.

Videbech P, Ravnkilde B (2004). Hippocampal volume and depression: a meta-analysis of MRI studies. Am J Psychiatry 161: 1957-1966.

Vollmayr B, Henn FA (2001). Learned helplessness in the rat: improvements in validity and reliability. Brain Res Protoc 8: 1-7.

Watanabe Y, Gould E, McEwen BS (1992). Stress induces atrophy of apical dendrites of hippocampal CA3 pyramidal neurons. Brain Res 588: 341-345.

World Health Organization (2001). The World Health Report 2001. http://www.who.int/mental_health/management/depression/defi nition/en/print.html.

$\mathrm{Xu} \mathrm{H}$, He J, Richardson JS, Li XM (2004). The response of synaptophysin and microtubule-associated protein 1 to restraint stress in rat hippocampus and its modulation by venlafaxine. J Neurochem 91: 1380-1388.

Zazpe A, Artaiz I, Labeaga L, Lucero ML, Orjales A (2007). Reversal of learned helplessness by selective serotonin reuptake inhibitors in rats is not dependent on 5-HT availability. Neuropharmacology 52: 975-984. 